\title{
Postural Rehabilitation for Adolescent Idiopathic Scoliosis during Growth
}

\author{
Hans-Rudolf Weiss ${ }^{1}$, Marc Michael Moramarco ${ }^{2}$, Maksym Borysov ${ }^{3}$, \\ Shu Yan $\mathrm{Ng}^{4}$, Sang Gil Lee ${ }^{5}$, Xiaofeng $\mathrm{Nan}^{6}$, Kathryn Ann Moramarco ${ }^{2}$ \\ ${ }^{1}$ Orthopedic Rehabilitation Services, Gensingen, Germany \\ ${ }^{2}$ Scoliosis 3DC, Woburn, MA, USA \\ ${ }^{3}$ Orttech-Plus Rehabilitation Services, Kharkov, Ukraine \\ ${ }^{4}$ Wanchai Chiropractic Clinic, Wanchai, Hong Kong \\ ${ }^{5}$ Power Schroth Corrective Exercise Center, Seoul, Korea \\ ${ }^{6}$ Nan Xiaofeng's Spinal Orthopedic Workshop, Xi'an Shanxi, China
}

Long-term follow-up of untreated patients with adolescent idiopathic scoliosis (AIS) indicates that, with the exception of some extremely severe cases, AIS does not have a significant impact on quality of life and does not result in dire consequences. In view of the relatively benign nature of AIS and the long-term complications of surgery, the indications for treatment should be reviewed. Furthermore, recent studies have shown that scoliosis-specific exercises focusing on postural rehabilitation can positively influence the spinal curvatures in growing adolescents. Experiential postural re-education is a conservative, non-invasive approach, and its role in the management of AIS warrants further study. This article reviews current evidence for the inclusion of various forms of postural reeducation in the management of AIS. Recent comprehensive reviews have been researched including a manual and PubMed search for evidence regarding the effectiveness of physical/postural re-education/physiotherapy programs in growing AIS patients. This search revealed that there were few studies on the application of postural re-education in the management of AIS. These studies revealed that postural re-education in the form of exercise rehabilitation programs may have a positive influence on scoliosis; however, the various programs were difficult to compare. More research is necessary. There is at present Level 1 evidence for the effectiveness of Schroth scoliosis exercises in the management of AIS. Whether this evidence can be extrapolated to include other forms of scoliosis-pattern-specific exercises requires further investigation. Because corrective postures theoretically reduce the asymmetric loading of the spinal deformities and reverse the vicious cycle of spinal curvature progression, their integration into AIS programs may be beneficial and should be further examined.

Keywords: Scoliosis; Physiotherapy; Rehabilitation; Treatment

\section{Introduction}

Adolescent idiopathic scoliosis (AIS) is defined as a three dimensional spinal deformity [1-4] with no identifiable causes $[5,6]$ in a growing child who is otherwise healthy.
On inspection, the child can be seen with trunk asymmetry, which is accentuated in forward bending. This is because the costal or lumbar hump becomes more prominent in this position. A "costal hump" evolves as the ribs on the thoracic concave side twist in a ventral direction.

Received Aug 11, 2015; Revised Oct 11, 2015; Accepted Oct 12, 2015

Corresponding author: Shu Yan $\mathrm{Ng}$

Wanchai Chiropractic Centre, 11/fl China Hong Kong Tower, 8 Hennessy Road, Wanchai, Hong Kong

Tel: +852-3998-3208, Fax: +852-3998-3222, E-mail: ngshuyanhcc@gmail.com 
A diagnosis of scoliosis is verified by an X-ray of the full spine with the patient in a standing position [7].

The reported rate of progression of AIS varies according to the study [8-10]. This may be due to differences in the samples studied and the definition of 'progression'. It is possible that a small percentage of those with minor distortions may resolve [8]. In contrast, Lonstein and Carlson [8] and Sahlstrand and Lidstrom [11] reported that curves of greater severity have a higher probability of progression. When considering comparable-sized curvatures, females are four to ten times more likely to experience progression, when compared to males [3,12]. As skeletal maturity increases, the risk of progression decreases. Yet, greater curves still tend to worsen after skeletal maturity $[7,13,14]$.

Since the causes of AIS are not clear, this has led to a variety of different treatment options. Up until recently, little attention has been paid to the role of postural rehabilitation in the management of AIS, despite the fact that it has been shown to be effective when integrated into scoliosisspecific exercises [15].

Treatment indications for scoliosis depend on the magnitude of the curvature at presentation and maturity of the patient $[3,4,7]$. Historically, in central Europe, treatment has consisted of the following protocols: (1) Observation in mild curvatures during growth. (2) Brace treatment in curvatures exceeding $20^{\circ}$ during growth. (3) Postural rehabilitation in moderate curvatures during growth and for curvatures exceeding $35^{\circ}$ after growth [4]. (4) Spinal fusion surgery.

In 1921, Katharina Schroth of Germany began to address the role of body mechanics for the treatment of scoliosis with an emphasis on rotational breathing [7]. Scoliosis patients display an asymmetric breathing pattern [16], which tends to rotate the thorax and aggravates spinal deformities. Breathing forces are directed towards the convexity of the spinal curvature, which results in increased trunk rotation. Regular deep breathing using the dysfunctional breathing pattern should be avoided [16]. Dysfunctional breathing patterns and incorrect postures during activities of daily living (ADL) have all been implicated in the pathogenesis of spinal curvatures [16]. They may be important as progression factors [17], particularly in AIS patients with Cobb angles in excess of $30^{\circ}$.

Similarly, posture may positively or negatively influence spinal curvatures [18]. Adoption of corrective postures (Table 1) during daily activities would avoid improper

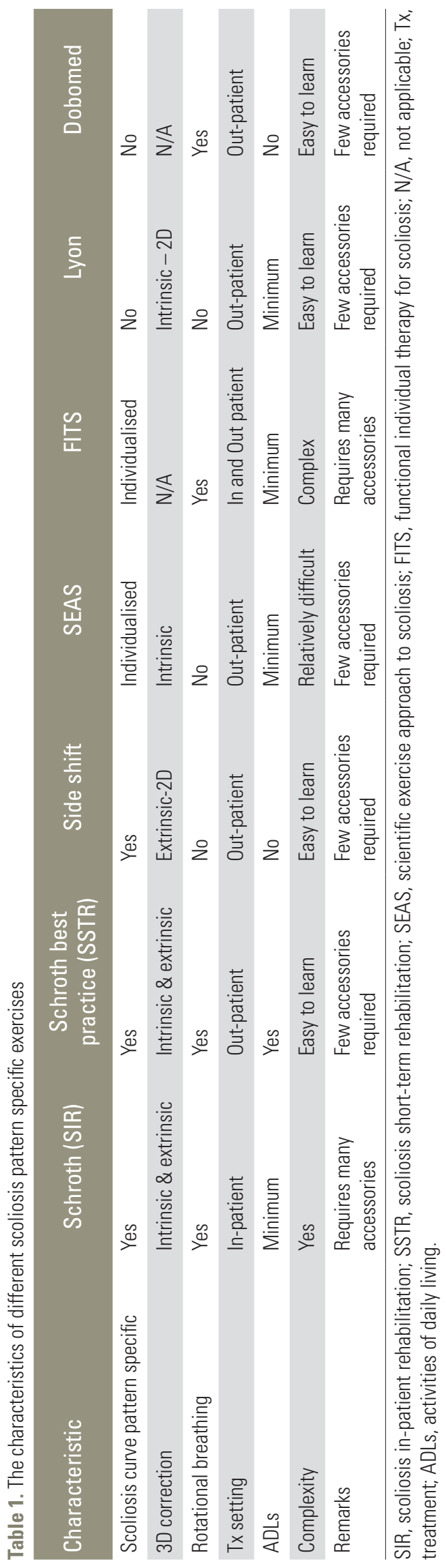


curve loading during the day. Incorrect postures during daily activities would increase asymmetric vertebral loading and growth in a scoliotic [19]. It is possible that for these reasons many practitioners instruct patients not to slump into habitual postures after performing scoliosis patterns specific exercises $[7,16]$. Failure of maintaining correct or corrective postures at rest may nullify all the efforts spent in performing the exercises [16].

Exercises and sports are beneficial to the overall health of individuals. Patients with AIS are encouraged to exercise regularly [17]. Despite this, certain activities may exacerbate spinal asymmetries. One example is a recent study that found that competitive swimming might increase spinal asymmetries and spinal curvatures [20]. The finding, though preliminary, is important in view of the fact that many physicians advise their scoliosis patients to swim to improve the back muscle strength and endurance, without realizing the possible risks. Owing to the possibility of improper postures causing progression of spinal curvatures, postural training has been integrated into certain scoliosis treatment programs. Here, we investigate the role and the effectiveness of various exercise-based programs and/or postural rehabilitation techniques in the management of AIS.

\section{Background}

\section{The role of posture in activities of daily living}

Some postures can influence the spinal curvatures positively or negatively, depending on the curve types. A kneeling movement may aggravate a thoracolumbar or lumbar curve, but reduce a thoracic curve [16]. On the contrary, contralateral kneeling movement may exaggerate the thoracic curve and reduce the thoracolumbar or lumbar curvatures (Table 1). Similarly, sitting with legs crossed on the floor may affect the spinal alignment positively or negatively [16]. Unfortunately, the importance of these clinical observations has not been studied, let alone the impact of scoliosis-curve-specific ADLs on the pathogenesis of scoliosis progression.

Extension, lateral flexion and rotation exercises [16] have been reported to be contraindicated in AIS. Back extension exercises reduce thoracic kyphosis. This may destabilize the thoracic spine, increase its propensity for lateral flexion and rotation, and increase the spinal curvature [21]. AIS patients, particularly those with double curves, should also avoid performing lateral flexion exercises [16]. Laterally flexing to one side reduces one of the curvatures, but increases the other. Swedish bending exercises that were advocated more than 50 years ago were found to cause progression of scoliosis in many cases [16]. Rotational exercises have also been reported to increase rib hump [16].

\section{Side-shift}

Depending on the curve types, patients are advised to side-shift to open up and relieve the pressure from the concavities of the curvature [7,16,22]. Gram and Hasan [18] found that AIS patients tend to lean towards the side of convexity. The lateral lean increases the overhang of the right thorax and could possibly increase the deformity of the spine. To counteract this, patients with right thoracic scoliosis are advised to shift towards the side of concavity to open up the thoracic concavity and to reduce the curvature during relaxed sitting $[7,16]$.

\section{Sitting postures}

School children spend many hours writing, reading, watching TV and doing homework on a daily basis. Their sitting postures are important, because improper sitting postures may result in progression of spinal curvatures [23]. Studies have shown that children habitually slumped or when were asked to relax. There was a reduction in lumbar lordosis [17,24] and thoracic kyphosis [24]. The reduction in thoracolumbar lordosis did not differ significantly between AIS patients and normal children [25] in relaxed sitting, erect sitting, or crossed-leg sitting.

When asked to sit in their habitual position, normal children aged 11-13 sat with a loss of lumbar lordosis [24]. The 19 AIS patients evaluated by Gram and Hasan [18] had a similar sitting pattern. Yet, van Loon et al. [26] found that AIS patients tended to sit with a more marked loss of thoracolumbar lordosis. This may be related to the marked neuromuscular tightness in AIS patients [27].

Though the spinal sagittal profile does not differ significantly between AIS adolescents and normal controls, there are marked differences in the frontal plane. When asked to sit relaxed, the majority of AIS children lean to one side [25]. Using infrared markers and a camera to study the sitting postures of 19 AIS patients, Gram and Hasan [18] found that the subjects tended to lean to one 
side when seated in a relaxed pose. The lean was curvetype dependent, differing in single and double scoliosis curves. In single thoracic and lumbar curves, the subject tends to lean laterally to the side of convexity. The lean which was defined as the horizontal distance between S1 and a perpendicular line from $\mathrm{C} 7$ in the frontal plane, averaged $1 \mathrm{~cm}$. The distance of the lean was smaller in double curves. In relaxed sitting, the lean was towards the concavity of the lumbar curve [17] in the presence of a double curve.

Similar findings were reported by Drza-Grabiec et al. [24]. They filmed 91 normal children aged 11-13 in their natural relaxed sitting postures, and found marked asymmetry in their relaxed sitting postures; also, the angular value of existing scoliosis increased after prolonged sitting [24]. AIS patients with thoracolumbar curvatures were reported to have increased scoliosis when sitting [25].

\section{Standing postures}

Standing is inherently associated with an increase in spinal curvature, as a result of gravitational forces. Clinical observation showed that AIS patients tend to bear weight on one side [16]. When standing, the spinal curvature increases when compared to relaxed and erect sitting [17].

\section{Carrying A backpack}

Regular carrying of schoolbags and backpacks can affect the spinal curvature. The effects depend on the mode of loading, the weight of the carriage, and the side of the loading. Carrying the bag on one shoulder, across the body and on the back affect the spine in different ways [28-32]. These findings are minimally important because children spend far less time carrying a backpack; however, they are conceptually important when considering the role that postural re-education has on the spinal configuration.

\section{Scoliotic breathing pattern}

Lehnert-Schroth [16] described the scoliotic breathing pattern. This asymmetric breathing pattern may be caused by faulty breathing mechanics. In right thoracic scoliosis, the right dorsal area is convex and the left is concave. Ventrally, the opposite is present. The right anterior chest is concave and the left anterior chest is convex. During breathing, forces are directed in the direction of convexi- ties, i.e., to the right posterior thoracic area and the left anterior thoracic area. This tends to rotate the thorax.

In thoracic scoliosis, the lung in the convex side is larger than the counterpart in the concave side, which is more compressed. With inhalation, which is an active process, air fills both lungs. The lung on the convex side, being larger and less compressed, tends to be more inflated than the lung on the concave side. During exhalation, which is a passive process, air is expelled from both lungs. As a result of increased chest wall elasticity, more air is expelled from the concave side. The results are that the size differential of the lung increases with each deep breath. There is a possibility that this dysfunctional breathing pattern, if performed regularly, may be a factor that contributes to increased rotation and progression of spinal curvature [16]. Rehabilitation of the scoliotic breathing pattern is an essential component of Schroth scoliosis exercise, one of the programs discussed in this review, which also incorporates postural re-education for the management of AIS.

\section{Materials and Methods}

PubMed searches were performed using the key words 'scoliosis, exercise, scoliosis-pattern-specific-exercises (SSE), physiotherapy, rehabilitation, active self-correction' to identify studies on the use of physical exercises, scoliosis-pattern-specific exercises and physiotherapy in the treatment of AIS. The studies are reviewed to ascertain if the treatment outcome support the use of scoliosispattern-specific exercises and postural rehabilitation in the management of AIS in growing children.

\section{Results}

Various studies have shown that scoliosis-pattern-specific exercises reduce Cobb angle in AIS patients [33-41] and angle of trunk rotation (ATR) [37,38,40,42-45]. Scoliosispattern-specific exercises (Table 2) have been found to be superior to general physiotherapy treatments in reducing spinal deformities [43,44,46]. Different scoliosis-patternspecific exercises have been shown to stabilize and improve spinal curves, when used alone or in combination with a brace. Functional Individual Therapy for Scoliosis (FITS) [39], Dobomed [47], side-shift [34], scientific exercise approach to scoliosis (SEAS) [37], Schroth [33,35] and Schroth Best Practice $[38,40,41]$ have all been shown 
Table 2. Some of the corrective postures that should be adopted during daily activities in patients with idiopathic scoliosis

\begin{tabular}{|c|c|c|}
\hline Activities of daily living ${ }^{\text {al }}$ & Position of the pelvis & Corrective movement \\
\hline \multicolumn{3}{|l|}{ Sitting } \\
\hline 4 Curve & Pelvis shifts to the side of thoracic convexity & $\begin{array}{l}\text { Lower the shifted pelvis (the side of thoracic convexity) and } \\
\text { translate the thoracic spine to side of concavity }\end{array}$ \\
\hline 3 Curve & Pelvis shifts to the side of thoracic concavity & $\begin{array}{l}\text { Lower the shifted pelvis (the side of thoracic concavity) and } \\
\text { translate the thoracic spine to side of concavity }\end{array}$ \\
\hline \multicolumn{3}{|l|}{ Standing } \\
\hline 4 Curve & Pelvis shifts to the side of thoracic convexity & $\begin{array}{l}\text { Lower the shifted pelvis (the side of thoracic convexity) and } \\
\text { translate the thoracic spine to side of concavity }\end{array}$ \\
\hline 3 Curve & Pelvis shifts to the side of thoracic concavity & $\begin{array}{l}\text { Lower the shifted pelvis (the side of thoracic concavity) and } \\
\text { translate the thoracic spine to side of concavity }\end{array}$ \\
\hline \multicolumn{3}{|l|}{ Kneeling } \\
\hline 4 Curve & - & $\begin{array}{l}\text { Lower the shifted pelvis and flex the contralateral hip and } \\
\text { knee }\end{array}$ \\
\hline 3 Curve & - & $\begin{array}{l}\text { Lower the shifted pelvis and flex the contralateral hip and } \\
\text { knee }\end{array}$ \\
\hline
\end{tabular}

alTypes of curves: (basing on Lehnert-Schroth Augmented Classification).

to stabilize and improve spinal curves in AIS patients.

A study, using FITS exercises on patients with AIS with an average age of 11.9 , Risser $0-2$ and single scoliosis curve measuring $17.7^{\circ}$, showed that $50 \%$ of the patients had the curves improved, $46.2 \%$ stabilized and $3.8 \%$ worsened [39]. For patients with double scoliosis, the outcome was similar though a smaller percentage of curves stabilized. In this group of patients, 50\% improved, $30.8 \%$ stabilized and $19.2 \%$ of the cases worsened [39].

Dobomed exercises, when used in combination with Chêneau bracing for an average period of $43 \pm 9$ months were found to stabilize progressive thoracic scoliosis in $89 \%$ of the AIS patients [48]. $11 \%$ of the patients progressed to surgery [48]. In an earlier study, the average Cobb angle of 152 AIS patients aged 14.2 years increased from $25.92^{\circ}$ to $31.04^{\circ}$ after a treatment period of 31.8 months [49].

Side-shift and hitch exercises which are specifically targeted to reduce thoracic, thoracolumbar and lumbar curves respectively by laterally shifting to the side of concavity were also shown to stabilize scoliotic curves, together with part-time bracing [34]. Of the 328 patients treated, 308 patients with an average age of 13.9 and initial Cobb angle of $31.2^{\circ}$ performed regular side-shift and hitch exercises, together with part time bracing whenever indicated, were followed up to the average age of 18.6. The Cobb angle at the conclusion of treatment was $32.6^{\circ}$ [34].

SEAS has also been shown to be able to stabilize mild scoliotic curves [37]. The treatment outcome of a group of AIS patients treated by SEAS was compared with that of the control group treated by general physiotherapy treatment provided by individual therapist. The average age of the treatment group was 12.7, with Cobb angle of $15.3^{\circ}$ and ATR of $8.9^{\circ}$, with the control group having similar age and curve severity. After performing SEAS.02 for one year, one patient $(4.3 \%)$ in the treatment group progressed and required surgery. In the control group, 5 patients (20\%) progressed to surgery. Based on a change of $5^{\circ} \mathrm{Cobb}$ angle as a reference for improvement or progression of the curvature, $28.9 \%$ of the patients in the treatment group improved, $68.4 \%$ were stable and $2.7 \%$ became worse. In the control group, 5\% improved, $82.1 \%$ were stable and $12.9 \%$ became worse. Basing on a change of $2^{\circ}$ ATR as a reference for improvement or progression of spinal curves, $30.4 \%$ improved in the treatment group, $56.5 \%$ were stable and $13.1 \%$ became worse. For the control group, $36.4 \%$ improved, $36.3 \%$ were stable and $27.3 \%$ became worse [37].

One randomized-controlled study compared the effects of active self-correction and task-oriented spinal exercises with general exercises in patients with mild AIS, with Cobb angle of less than $25^{\circ}$ [43]. Outcome measures one year later showed that the training in the intervention group led to a significant improvement (decrease in Cobb angle of $5^{\circ}$ ), whereas the control group remained stable [43]. Thus scoliosis-pattern-specific exercises are superior 
to traditional exercises, which are unspecific exercises in reducing spinal deformities in patients with mild AIS [43].

Schroth scoliosis in-patient rehabilitation (SIR) has been used in the treatment of AIS for many decades $[16,35]$. SIR was found to improve curvatures of patients with AIS [33]. One hundred and seven patients with AIS, with an average Cobb angle of $43.06^{\circ}$, were treated by an in-patient exercise program. After 4-6 weeks of treatment, the average Cobb angle reduced to $38.96^{\circ}$. An improvement of $5^{\circ}$ or more was found in $43.93 \%$ of the patients, $53.27 \%$ were unchanged and in $2.8 \%$ the curve increased by $5^{\circ}$ or more. The improvement in Cobb angle was highly significant [33]. Schroth scoliosis in-patient rehabilitation was also found to significantly reduce the progression risk of patients as compared to age and sex-matched controls [35]. Also, SIR was also found to reduce right heart stress [50], improve vital capacity [45] and improve muscle balance in patients with AIS [51].

A cohort study in Turkey similarly showed improvement in Cobb angle, vital capacity and muscle strength of patients with AIS, undergoing Schroth therapy on an outpatient basis [36]. Fifty patients with an average age of $14.15 \pm 1.69$ were instructed to perform 4 hours of exercises every day for 5 days consecutively for 6 weeks. They were thereafter advised to perform the Schroth exercises at home. After one year of the exercises, the Cobb angle reduced from an average of $26.1^{\circ}$ to $17.85^{\circ}$ [36]. The vital capacity increased by $15 \%$ in one year. There was an increase in muscle strength and recovery of postural defects in all patients [36].

The increase in back muscle endurance has recently been confirmed by a randomized-controlled study, which compared one group of AIS patients treated by Schroth exercises in addition to standard care with another group of patients treated by standard care alone [52]. Addition of Schroth exercises significantly increased the back muscle endurance and reduced the SRS-22r pain score [52] at different follow-up periods.

Noh et al. [46] compared a novel spinal corrective technique (CST), based on Schroth exercises and core stabilization techniques with conventional physiotherapy exercises in the management of AIS. CST resulted in greater improvement in Cobb angle and vertebral rotation when compared to general exercises in the group of patients aged between 10-19. Also, CST improved the pelvic incidence to normal range. Yet, the method did not improve the sagittal profile [46].
The effectiveness of Schroth exercises in AIS was reported in another randomized controlled trial [44]. Fortyfive patients with AIS were randomized into three groups, each with 15 patients. The intervention group performed Schroth exercises under physiotherapist supervision for 6 weeks and continued to do the exercises at home. The second group did the Schroth exercises at home, after they were taught the exercises by a physiotherapist. The third group was treated by observation. At the conclusion of the study at 24 weeks, evaluation showed that the Cobb angle and angle of trunk rotation improved significantly in the intervention group, when compared with the other two groups [44]. This suggested that scoliosis-pattern-specific exercises are superior to observation in the treatment of AIS [44].

The newest branch of Schroth, Schroth Best Practice, was introduced in an attempt to improve the time efficiency of rehabilitation [15]. Furthermore, postural reeducation was integrated in the form of ADL training into the Schroth Best Practice program, something that was not a major component of the original Schroth treatment program [15]. The addition of ADLs is important to ensure that postural control is not lost throughout the day. Without integrating the corrective ADLs, the spinal curve(s) continues to be loaded asymmetrically during daily activities, making improvement via exercise more difficult [15]. Another addition to the program focused on maintaining or improving the thoracolumbar natural curvature. This was found to decrease the lateral deviation of the trunk as well [53]. A pilot investigation showed that four days of treatment could reduce the ATR significantly [38] in patients with an average age of 14 and average Cobb angle of $46^{\circ}$. The ATR reduced from $10.3^{\circ}$ to $8.2^{\circ}$ after 4 days of treatment. The improvement in outcome is similar to that which can be achieved in 3-4 weeks of inpatient rehabilitation. This was subsequently confirmed by two studies $[40,42]$. In additional studies, Schroth Best Practice has been shown to reduce Cobb angle [42] and other signs and symptoms of AIS [41].

\section{Discussion}

There have been many studies proving that scoliosis-pattern-specific-exercises improve or stabilize scoliotic curves in patients with AIS [37-42]. A systematic review in 2008 concluded that SSE was effective in the treatment of AIS [54]. A Cochrane review in 2012, however, concluded that 
there was insufficient evidence in support of the use of exercises in the treatment of AIS [55]. Nevertheless, since the publication of the review, four controlled outcome papers on the effects of scoliosis-specific exercises on AIS have been published $[43,44,46,52]$. Three were randomized controlled trials $[43,44,52]$ and one was a controlled trial [43]. The significance of this, collectively, is that there is a proactive approach to improving scoliosis, beginning as early as the observation phase.

When scoliosis progresses to a Cobb angle of or exceeding $45^{\circ}-50^{\circ}$ in growing children, spinal fusion surgery is often recommended. This is because curves greater than $50^{\circ}$ at skeletal maturity are reported to progress an additional $20^{\circ}-30^{\circ}$ over the subsequent $30-40$ years period $[56,57]$. Despite this, a long-term follow-up study of untreated patients with AIS has shown that the consequences of AIS over a lifetime are minimal, sometimes moderate in more severe cases, and very rarely life threatening $[3,4,7,14,56]$. Recently, a study showed that the SRS$22 \mathrm{r}$ scores of non-operated and operated groups of AIS patients were similar, which implied limited benefits of surgery [58]. In view of the relative benign nature of AIS [58] and the long-term complications of surgery [59-61], it may be necessary to review the current indications for treatment of AIS.

It is evident that there are many different approaches to the treatment of AIS. Not all scoliosis exercises are equally effective [62]. The principles of their correction are different. Some use general exercises without a defined correction procedure (Dobomed), while others mainly aim at specific curvature correction (Fig. 1) (side-shift, self-correction exercises, SEAS, Schroth, and Schroth Best Practice). Also, because the demographics and curve severity of study populations and the outcome measures have been different, direct comparison of the effectiveness of the varied approaches is impossible.

Among these methods, the Schroth approach is the only one that has been proven to be effective in three controlled trials $[44,46,52]$, two of which were randomized [44,52]. An updated version, Schroth Best Practice, evolved out of the original Schroth method to provide an all-encompassing postural rehabilitation program for

\section{Augmented Lehnert-Schroth (ALS) Classification}


From left to right: $3 \mathrm{CH}$ (3-curve with hip prominence), 3CTL (3-curve with hip prominence thoracolumbar), 3C (3-curve balanced), 3CL (3-curve with long lumbar countercurve), 4C (4-curve double), 4CL (4-curve single lumbar), 4CTL (4-curve single thoracolumbar).

Fig. 1. The Augmented Lehnert-Schroth classification as used for postural rehabilitation and for scoliosis pattern specific brace adjustment as well. From Weiss et al. [7], with kind permission from Lambert Academic Publishing. 
the management of AIS. The exercise approach has been simplified (Fig. 2) with added incorporation of sagittal plane correction and scoliosis-pattern-specific activities to daily living (ADLs) (Fig. 3) into the program [38]. Sagittal plane correction has been found to decrease the lateral deviation of the trunk as well [53]. Of particular significance is the incorporation of ADLs (Figs. 3, 4) in the management of AIS, as it was reasoned that thirty minutes of SSE daily is unlikely to improve curvature if the patient does not adopt proper posture during daily activities. Training scoliosis patients on how to avoid loss of postural control during everyday activities can improve outcomes. This is because scoliosis curve-pattern-specific ADLs take into account the pelvic shift and the types of scoliosis curves to unload the curve. Without integrating the corrective ADLs, the curve(s) are loaded during the course of the day making improvement via exercise more difficult [7].

Studies have shown that this simplified approach (Schroth Best Practice) does not compromise the outcome of the exercise program $[42,44]$. These updated methods of postural re-education (Fig. 4) offer the potential for improvement above and beyond the original Schroth program [42]. They also address other signs and symptoms of scoliosis as recognized in independent studies [40-42]. Patients are also able to achieve comparable results within one week or less, as opposed to the longer durations with traditional in-patient rehabilitation [38]. In the study by Lee [42], in the subgroup of patients with larger curvatures $\left(>30^{\circ}\right)$, the Cobb angle corrected about twice as much as during in-patient rehabilitation in a comparable sample of patients.

Although there is Level 1 evidence for the Schroth approach and active self-correction, the evidence cannot be extrapolated to include every type of SSE, as the principle and dosage of each treatment approach differ. Studies need to be conducted to compare the effectiveness of different approaches using cohorts of similar demographics, curve severity and outcome measures, before the Level 1 evidence can properly be assigned to different exercise approaches.

Schroth Best Practice evolved out of the original Schroth program and includes principles of the original Schroth approach, as well as task-oriented exercises and active self-correction [43]. Since the outcomes are comparable or better than the original Schroth method, this program can be regarded as having Level 1 evidence.

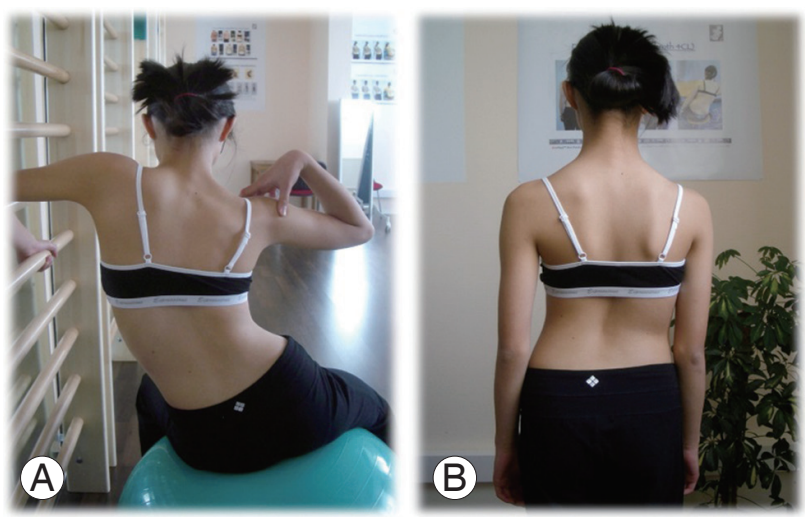

Fig. 2. Correcting exercise position (A) for a functional 3-curve pattern (see below) in comparison to the relaxed scoliotic posture (B). From Weiss et al. [7], with kind permission from Lambert Academic Publishing.
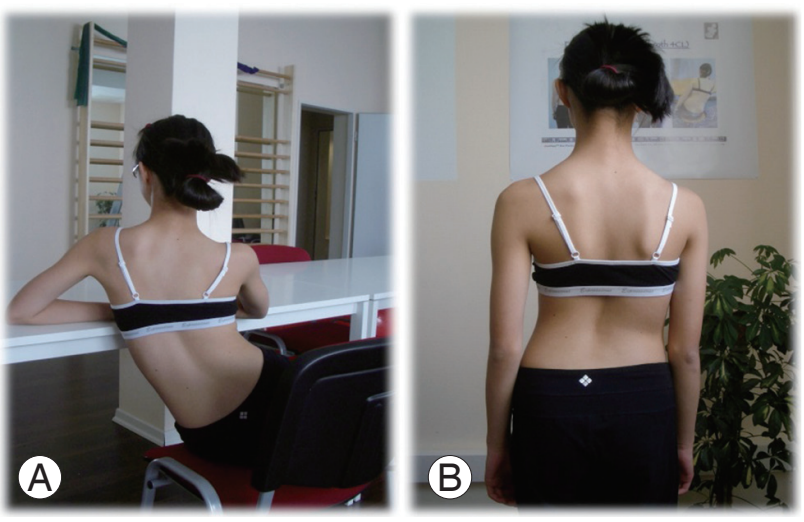

Fig. 3. Correcting ADL position (A) for a functional 3-curve pattern in comparison with the relaxed scoliotic posture (B). From Weiss et al. [7], with kind permission from Lambert Academic Publishing.
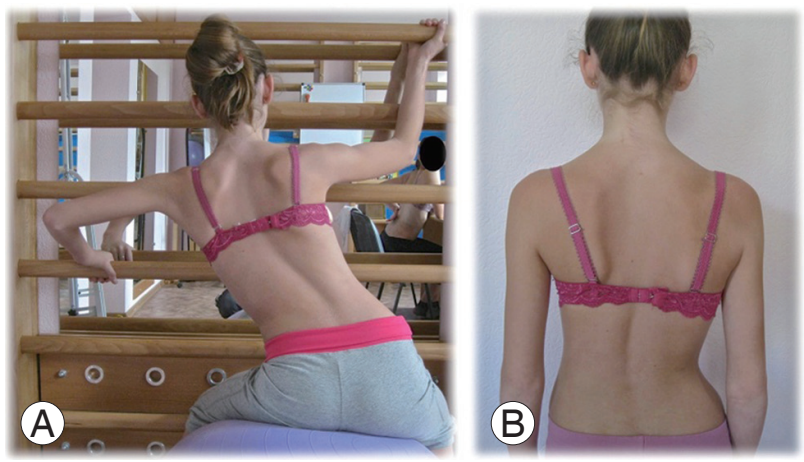

Fig. 4. Correcting exercise position (A) for a functional 4-curve pattern in comparison with the relaxed scoliotic posture (B). From Weiss et al. [7], with kind permission from Lambert Academic Publishing.

\section{Limitation}

The three independent studies on Schroth Best Practice have their limitations. Two of the papers [40,42] were 
prospective short-term cohort studies. The studies included patients who were relatively skeletally mature. The average age of patients in one study [40] was 13.7 years of age and that of another [42] was 16 years of age. Also, the period of intervention was short. One study involved exercising for 7 days [37] and another for 2.9 months [42]. Also, there was no follow-up of the patients until 5 years after skeletal maturity. The third study [41] evaluated patients before and after 6 weeks of the exercises using optical topography, electromyography and stabilometry. Whether the improvement in outcome can be maintained over a longer period of time, however, has not been investigated. Also, whether adoption of corrective postures in daily activities does reduce the asymmetrical loading of the scoliotic spines and the resultant spinal deformities remain to be studied. Thus while Schroth Best Practice should be incorporated in clinical practice, further studies are required to validate its individual components.

\section{Conclusions}

Postural rehabilitation using scoliosis-specific exercise can be effective in the treatment of AIS patients when incorporated into daily life. For the benefit of the patient, it should be integrated into the existing management of the condition. Schroth scoliosis exercises and active selfcorrection have both been validated by Level 1 evidence. Whether this evidence can be extrapolated to include other forms of scoliosis-pattern-specific exercises requires further investigation.

\section{Conflict of Interest}

HR Weiss is receiving financial support for attending symposia and receives royalties from Koob GmbH \& Co KG. The company is held by the spouse of HR Weiss.

None of the other authors report any competing interest or potential conflict of interest.

\section{References}

1. Heine J, Meister R. Quantitative analysis of the functions of the lungs and of blood-gases in young patients with scoliosis, using a "functionaldiagnostic minimal program”. Z Orthop Ihre Grenzgeb 1972; 110:56-62.

2. Meister R. Atemfunktion und Lungenkreislauf bei thorakaler Skoliose. Stuttgart [DE]: Georg Thieme Verlag; 1980.

3. Asher MA, Burton DC. Adolescent idiopathic scoliosis: natural history and long term treatment effects. Scoliosis 2006;1:2.

4. Weiss HR, Moramarco M. Scoliosis: treatment indications according to current evidence. OA Musculoskeletal Med 2013;1:1.

5. Heine J. Die Lumbalskoliose: eine Untersuchung zur Klinik und Prognose der Erkrankung. Stuttgart: F. Enke; 1980.

6. Perdriolle R, Vidal J. Thoracic idiopathic scoliosis curve evolution and prognosis. Spine (Phila Pa 1976) 1985;10:785-91.

7. Weiss HR, Lehnert-Schroth C, Moramarco M. Schroth therapy: advancements in conservative scoliosis treatment. Saarbruecken: Lambert Academic Publishing LAP; 2015.

8. Lonstein JE, Carlson JM. The prediction of curve progression in untreated idiopathic scoliosis during growth. J Bone Joint Surg Am 1984;66:1061-71.

9. Wiemann JM, Shah SA, Price CT. Nighttime bracing versus observation for early adolescent idiopathic scoliosis. J Pediatr Orthop 2014;34:603-6.

10. Danielsson AJ, Hasserius R, Ohlin A, Nachemson AL. A prospective study of brace treatment versus observation alone in adolescent idiopathic scoliosis: a follow-up mean of 16 years after maturity. Spine (Phila Pa 1976) 2007;32:2198-207.

11. Sahlstrand T, Lidstrom J. Equilibrium factors as predictors of the prognosis in adolescent idiopathic scoliosis. Clin Orthop Relat Res 1980;(152):232-6.

12. Weinstein SL, University of I, University of Iowa Health C, Virtual H. Adolescent idiopathic scoliosis prevalence, natural history, treatment indications. Iowa: University of Iowa; 1992.

13. Edgar MA. The natural history of unfused scoliosis. Orthopedics 1987;10:931-9.

14. Weinstein SL, Dolan LA, Spratt KF, Peterson KK, Spoonamore MJ, Ponseti IV. Health and function of patients with untreated idiopathic scoliosis: a 50-year natural history study. JAMA 2003;289:559-67.

15. Weiss HR, Hollaender M, Klein R. ADL based scoliosis rehabilitation: the key to an improvement of time-efficiency? Stud Health Technol Inform 2006; 123:594-8.

16. Lehnert-Schroth C. Three-dimensional treatment for 
scoliosis: physiotherapeutic method for deformities of the spine. Palo Alto, CA: Martindale Press; 2007.

17. Negrini S, Aulisa AG, Aulisa L, et al. 2011 SOSORT guidelines: Orthopaedic and Rehabilitation treatment of idiopathic scoliosis during growth. Scoliosis 2012; $7: 3$.

18. Gram MC, Hasan Z. The spinal curve in standing and sitting postures in children with idiopathic scoliosis. Spine (Phila Pa 1976) 1999;24:169-77.

19. Stokes IA, Burwell RG, Dangerfield PH. Biomechanical spinal growth modulation and progressive adolescent scoliosis: a test of the 'vicious cycle' pathogenetic hypothesis: summary of an electronic focus group debate of the IBSE. Scoliosis 2006;1:16.

20. Zaina F, Donzelli S, Lusini M, Minnella S, Negrini S. Swimming and spinal deformities: a cross-sectional study. J Pediatr 2015;166:163-7.

21. Rigo M, Quera-Salva G, Villagrasa M. Sagittal configuration of the spine in girls with idiopathic scoliosis: Progressing rather than initiating factor. In: Uyttendaele D, Dangerfield P, International Research Society of Spinal D, Meeting, editors. Research into spinal deformities 5. Amsterdam: IOS Press; 2006. p.90-4.

22. Maruyama T, Takeshita K, Kitagawa T. Side-shift exercise and hitch exercise. Stud Health Technol Inform 2008;135:246-9.

23. Czupryna K, Nowotny-Czupryna O, Nowotny J. Neuropathological aspects of conservative treatment of scoliosis: a theoretical view point. Ortop Traumatol Rehabil 2012;14:103-14.

24. Drza-Grabiec J, Snela S, Rykala J, Podgorska J, Rachwal M. Effects of the sitting position on the body posture of children aged 11 to 13 years. Work 2015;51:855-62.

25. Smidt GL, Van Meter SE, Hartman MD, Messaros SE, Rubsam DL, Anderson Welk K. Spine configuration and range of motion in normals and scoliotics. Clin Biomech (Bristol, Avon) 1994;9:303-9.

26. van Loon PJ, Kuhbauch BA, Thunnissen FB. Forced lordosis on the thoracolumbar junction can correct coronal plane deformity in adolescents with double major curve pattern idiopathic scoliosis. Spine (Phila Pa 1976) 2008;33:797-801.

27. van Loon PJ. Clinical detectable tension in the growing body: new and revisited signs in clinical examination in children with postural problems and spinal deformities. Restoration of lordosis on the thoracolumbar junction can correct sagittal and coronal plane deformity; a new (revisited) linked approach on the treatment and etiology of adolescent spinal deformities. Stud Health Technol Inform 2008;140: 52-8.

28. Bettany-Saltikov J, Warren J, Stamp M. Carrying a rucksack on either shoulder or the back, does it matter? Load induced functional scoliosis in "normal" young subjects. Stud Health Technol Inform 2008; 140:221-4.

29. O'Shea C, Bettany-Saltikov JA, Warren JG. Effect of same-sided and cross-body load carriage on 3D back shape in young adults. In: Uyttendaele D, Dangerfield P, International Research Society of Spinal D, Meeting, editors. Research into spinal deformities 5. Amsterdam, Netherlands; Washington, DC: IOS Press; 2006. p.159-63.

30. Chow DH, Kwok ML, Cheng JC, et al. The effect of backpack weight on the standing posture and balance of schoolgirls with adolescent idiopathic scoliosis and normal controls. Gait Posture 2006;24:173-81.

31. Hung-Kay Chow D, Kit-Fong Hin C, Ou D, Lai A. Carry-over effects of backpack carriage on trunk posture and repositioning ability. Int J Ind Ergon 2011;41:530-5.

32. Sahli S, Rebai H, Ghroubi S, Yahia A, Guermazi M, Elleuch MH. The effects of backpack load and carrying method on the balance of adolescent idiopathic scoliosis subjects. Spine J 2013;13:1835-42.

33. Weiss HR. Influence of an in-patient exercise program on scoliotic curve. Ital J Orthop Traumatol 1992;18:395-406.

34. Maruyama T, Kitagawa T, Takeshita K, Mochizuki K, Nakamura K. Conservative treatment for adolescent idiopathic scoliosis: can it reduce the incidence of surgical treatment? Pediatr Rehabil 2003;6:215-9.

35. Weiss HR, Weiss G, Petermann F. Incidence of curvature progression in idiopathic scoliosis patients treated with scoliosis in-patient rehabilitation (SIR): an age- and sex-matched controlled study. Pediatr Rehabil 2003;6:23-30.

36. Otman S, Kose N, Yakut Y. The efficacy of Schroth's 3-dimensional exercise therapy in the treatment of adolescent idiopathic scoliosis in Turkey. Neurosciences (Riyadh) 2005;10:277-83.

37. Negrini S, Negrini A, Romano M, Verzini N, Negrini 
A, Parzini S. A controlled prospective study on the efficacy of SEAS.02 exercises in preventing progression and bracing in mild idiopathic scoliosis. Stud Health Technol Inform 2006;123:523-6.

38. Weiss HR, Seibel S. Scoliosis short-term rehabilitation (SSTR): a pilot investigation. Internet J Rehabil 2009;1. http://dx.doi.org/10.5580/e71.

39. Bialek M. Conservative treatment of idiopathic scoliosis according to FITS concept: presentation of the method and preliminary, short term radiological and clinical results based on SOSORT and SRS criteria. Scoliosis 2011;6:25.

40. Borysov M, Borysov A. Scoliosis short-term rehabilitation (SSTR) according to 'Best Practice' standardsare the results repeatable? Scoliosis 2012;7:1.

41. Pugacheva N. Corrective exercises in multimodality therapy of idiopathic scoliosis in children - analysis of six weeks efficiency - pilot study. Stud Health Technol Inform 2012;176:365-71.

42. Lee SG. Improvement of curvature and deformity in a sample of patients with Idiopathic Scoliosis with specific exercises. OA Musculoskeletal Med 2014;2:6.

43. Monticone M, Ambrosini E, Cazzaniga D, Rocca B, Ferrante S. Active self-correction and task-oriented exercises reduce spinal deformity and improve quality of life in subjects with mild adolescent idiopathic scoliosis. Results of a randomised controlled trial. Eur Spine J 2014;23:1204-14.

44. Kuru T, Yeldan I, Dereli EE, Ozdincler AR, Dikici F, Colak I. The efficacy of three-dimensional Schroth exercises in adolescent idiopathic scoliosis: a randomised controlled clinical trial. Clin Rehabil 2016;30:181-90.

45. Weiss HR. The effect of an exercise program on vital capacity and rib mobility in patients with idiopathic scoliosis. Spine (Phila Pa 1976) 1991;16:88-93.

46. Noh DK, You JS, Koh JH, et al. Effects of novel corrective spinal technique on adolescent idiopathic scoliosis as assessed by radiographic imaging. J Back Musculoskelet Rehabil 2014;27:331-8.

47. Wnuk B, Durmala J, Dzierzega J, Dybula K, Dybula S, Wadolowski K. Short-term effects by using additional methods in DOBOMED preparation phase for AIS double major patients: pilot study. Scoliosis 2012; 7:O58

48. Durmala J, Kotwicki T, Piotrowski J. Stabilization of progressive thoracic adolescent idiopathic scoliosis using brace treatment and DoboMed physiotherapy. Scoliosis 2009;4:O29.

49. Dobosiewicz K, Durmala J, Czernicki K, Piotrowski J. Radiological results of Dobosiewicz method of threedimensional treatment of progressive idiopathic scoliosis. Stud Health Technol Inform 2006;123:267-72.

50. Weiss HR, Bickert W. Improvement of the parameters of right-heart stress evidenced by electrocardiographic examinations by the in-patient rehabilitation program according to Schroth in adult patients with scoliosis. Orthop Prax 1996;32:450-3.

51. Weiss HR. Imbalance of electromyographic activity and physical rehabilitation of patients with idiopathic scoliosis. Eur Spine J 1993;1:240-3.

52. Schreiber S, Parent EC, Moez EK, et al. The effect of Schroth exercises added to the standard of care on the quality of life and muscle endurance in adolescents with idiopathic scoliosis-an assessor and statistician blinded randomized controlled trial: "SOSORT 2015 Award Winner”. Scoliosis 2015;10:24.

53. Weiss HR, Klein R. Improving excellence in scoliosis rehabilitation: a controlled study of matched pairs. Pediatr Rehabil 2006;9:190-200.

54. Negrini S, Fusco C, Minozzi S, Atanasio S, Zaina F, Romano M. Exercises reduce the progression rate of adolescent idiopathic scoliosis: results of a comprehensive systematic review of the literature. Disabil Rehabil 2008;30:772-85.

55. Romano M, Minozzi S, Bettany-Saltikov J, et al. Exercises for adolescent idiopathic scoliosis. Cochrane Database Syst Rev 2012;8:CD007837.

56. Weinstein SL, Ponseti IV. Curve progression in idiopathic scoliosis. J Bone Joint Surg Am 1983;65:44755.

57. Ascani E, Bartolozzi P, Logroscino CA, et al. Natural history of untreated idiopathic scoliosis after skeletal maturity. Spine (Phila Pa 1976) 1986;11:784-9.

58. Ward WT, Roach JW, Frel N, Kenkre TS, Brooks MM. SRS-22r scores in non operated AIS patients with curve $\geqq 40^{\circ}$. Proceeding sof the Scoliosis Research Society 50th Annual Meeting and Course; 2015 Sep 30-Oct 3; Minneapolis, USA.

59. Westrick ER, Ward WT. Adolescent idiopathic scoliosis: 5-year to 20-year evidence-based surgical results. J Pediatr Orthop 2011;31:S61-8.

60. Weiss HR, Moramarco M, Moramarco K. Risks and long-term complications of adolescent idiopathic 
scoliosis surgery versus non-surgical and natural history outcomes. Hard Tissue 2013;2:27.

61. Weiss HR. Adolescent idiopathic scoliosis (AIS): an indication for surgery? A systematic review of the literature. Disabil Rehabil 2008;30:799-807.
62. Negrini S, Antonini G, Carabalona R, Minozzi S. Physical exercises as a treatment for adolescent idiopathic scoliosis: a systematic review. Pediatr Rehabil 2003;6:227-35. 